Kansas State University Libraries

New Prairie Press

\title{
A CLASSIFICATION OF UNREPLICATED FACTORIAL EXPERIMENTS FOR USE WITH THE ANALYSIS OF DETERMINISTIC SIMULATION MODELS
}
J L. Willers
B. T. Vinyard

Follow this and additional works at: https://newprairiepress.org/agstatconference

Part of the Agriculture Commons, and the Applied Statistics Commons

\section{(c) (1) $\Theta$}

This work is licensed under a Creative Commons Attribution-Noncommercial-No Derivative Works 4.0 License.

\section{Recommended Citation}

Willers, J L. and Vinyard, B. T. (1995). "A CLASSIFICATION OF UNREPLICATED FACTORIAL EXPERIMENTS FOR USE WITH THE ANALYSIS OF DETERMINISTIC SIMULATION MODELS," Conference on Applied Statistics in Agriculture. https://doi.org/10.4148/2475-7772.1337

This is brought to you for free and open access by the Conferences at New Prairie Press. It has been accepted for inclusion in Conference on Applied Statistics in Agriculture by an authorized administrator of New Prairie Press. For more information, please contact cads@k-state.edu. 


\title{
A CLASSIFICATION OF UNREPLICATED FACTORIAL EXPERIMENTS FOR USE WITH THE ANALYSIS OF DETERMINISTIC SIMULATION MODELS
}

\author{
J. L. Willers \\ USDA, ARS, Crop Simulation Research Unit \\ P.O. Box 5367 \\ Mississippi State, Mississippi 39762 \\ B. T. Vinyard \\ USDA, ARS, Southern Regional Research Center \\ 1100 Robert E. Lee Blvd \\ New Orleans, Louisiana 70124
}

\begin{abstract}
$\underline{\text { Abstract }}$
Deterministic simulation models are important in agricultural applications and their use is becoming increasingly common. Therefore, statistical procedures that interpret the output and evaluate the performance of deterministic models are necessary. The fact that deterministic computer simulation experiments cannot be replicated provides opportunities for using several procedures applicable to unreplicated factorial experiments. We discuss a classification scheme that selects the correct technique for most deterministic simulation experiments. The value of these techniques is their capability to estimate the experimental error variance for unreplicated computer experiments. Using these estimates of error, model developers and practitioners can more thoroughly analyze their deterministic simulation experiments.
\end{abstract}

Keywords: deterministic simulation models, simulation experiments, unreplicated factorials, key to classification, statistical analysis, estimation of error.

\section{Introduction}

There are many types of computer simulation models; however, in this paper, we use two broad groupings: stochastic and deterministic models. According to Law and Kelton (1991), a stochastic model is probabilistic (i.e., random components are present). As a consequence, the output is not predictable from run to run when the same specification of controllable input variables (Panis et al., 1994) and relationships are used. The output obtained from any given simulation run must be treated as only an estimate of the true characteristics of the model. On the other hand, a deterministic model has no probabilistic components; therefore, the output is "determined", or known exactly every time the same specification of controllable input variables and relationships are utilized. Once the model has been run, the response is predictable for every subsequent run that uses the same specific set of inputs (Law and Kelton, 1991). 
However, it is true that the response for a deterministic model cannot be predicted (except for a very simplistic one) before it is observed. In this sense, a deterministic model can be construed as having "random" behavior. Since it is not possible a priori to predict the response of a complex, deterministic model, it is necessary to have analysis methods that interpret the output for different sets of inputs. However, the nature of the input can be quite diverse and the method of statistical analysis used is dependent upon how inputs are contrived. Therefore, the purpose of this paper is to provide guidelines on selecting an analysis technique driven by the characteristics of the input; i.e., the input specifies the treatment structure of the deterministic simulation experiment.

The methods used to create this classification have been applied to the analysis of nonreplicated factorial experiments in industrial or academic settings. The texts of Box, Hunter, \& Hunter (1978), Daniel (1976), and Milliken and Johnson (1989) comprise the primary literature used to develop the classification scheme described below. The main purpose of the paper is to help select an analysis method, not discuss in detail the analysis method itself. Readers should refer to the cited literature to find further information on a particular method of analysis.

\section{Presentation of the Classification}

We discuss a classification scheme, known as a key, that selects the correct factorial technique for the analysis of most deterministic simulation experiments. The selection process is guided by the characteristics of the model inputs, or factors. Typically, a key is a tool used in the biological sciences to determine the taxonomic classification (i.e., name) of an unfamiliar biological organism; generally, its order, family, genus and species. For example, the common dandelion belongs to order-Sympetalae, family-Compositae, genus-Taraxacum, and is the species-officinale. A key is a collection of numbered couplets. Each couplet typically presents two mutually exclusive alternatives assigned a different letter of either "a" or "b". Upon selection of one alternative, guidance is provided to another set of numbered couplets that describe other attributes used to distinguish between alternatives. One proceeds through the key until an alternative becomes specific enough to name the organism. It is possible in a key to have some alternatives key out more than once. One reference that can be consulted on the development and use of a key is Porter (1967).

It is important to have a key that can be used to select a method of analysis for deterministic simulation experiments, because the characteristics of controllable input variables or relationships can be diverse. Given the numerous possibilities for specifying inputs to deterministic models it is useful to have guidelines to determine which analysis techniques are most appropriate. A second important consideration that motivates the development of a key is the fact that deterministic simulation experiments cannot be replicated To see why these experiments cannot be replicated, consider the following points. For many experiments, replication is important because experimental units have the "... property of producing different 
results even when subjected to the same treatment", and as a consequence, "... these differences, whether large or small, contribute to the experimental error..." (Cochran \& Cox, 1957). This concept is an important principle and for any experiment, it is always useful to clearly know what the experimental units are. With computer experiments, it is most useful to consider the model itself, as represented by code, to be the experimental unit. Thus, a stochastic model will exhibit variability among runs using the same treatments as input. However, deterministic models comprise a set of unique experimental units in that they do not exhibit variability when subjected numerous times to the same treatment. A consequence is that unique methods need to be used to estimate the experimental error variance (e.g., half-normal plotting, etc.).

The viewpoint that the simulation model is the experimental unit means that controllable input variables and/or relationships define the treatments applied to the experimental unit. For computer experiments, factors are equivalent to the input parameters and structural assumptions of the model (Law and Kelton, 1991). These factors can be quantitative, qualitative, controllable or, at times, uncontrollable. Law and Kelton (1991) present a good discussion of experimental design in the context of simulation experiments.

In the key (Table 1) presented here, each alternative within a couplet will end in either an acronym, number, or reference. Acronyms are more fully defined at the end of the key and are the name of the specific factorial analysis that can be used. Accompanying the key are short narratives of each particular factorial experiment that is designated by an acronym. These brief descriptions in the text provide additional references that can be consulted.

The same information in Table 1 has been drawn as a decision tree in Figure 1 ( $a \& b$ ). Some users may find this presentation easier to use. However, presenting the information of a key in decision tree form becomes difficult and more cumbersome if numerous couplets exist; therefore, it is not possible to draw a simple decision tree for all keys.

\section{Comments on the Key.}

In this section, brief comments are provided on each couplet. Whenever a couplet identifies an analysis procedure, the corresponding narrative will provide primary references that can be consulted for further detail. Reference is also provided to where software can be found that performs the analysis. Where available, mention will be made to a paper that used that particular analysis for a simulation experiment.

If an alternative ends with the acronym, "CE (cutting edge)", this means either that no known factorial analysis is possible, or refers to the analysis of a simulation experiment context not yet tried by the authors.

Couplet 1. The classification scheme, or key, quickly excludes stochastic simulation experiments because it is possible to replicate these experiments. Since replication is possible, 
these experiments can be analyzed using traditional principles of experimental design (Law and Kelton, 1991; Shannon, 1975; Swartzman and Kaluzny, 1987) and are not discussed further. The focus of the paper is instead on the selection of an analysis method for a deterministic simulation experiment.

Couplet 2. If the simulation experiment has strong correlations in time, or has characteristics of a repeated measures design, the use of unreplicated, factorial procedures may not be appropriate. Therefore, whenever a series of observations constitute the model output, one should consult Garratt (1975). He discusses several techniques that might apply to this situation. Also, Wallach and Goffinet (1989) describe the use of the mean squared error of prediction (MSEP) in several different situations. This statistic might be useful when the output is strongly correlated. But, if only one point comprises the output, or the possibility of time correlated output is remote (e.g., observations are a year or more apart) then continue to select an analysis procedure.

Couplet 3. This couplet uses the number of levels of the factors to begin sorting out the procedures; continue the process by branching to couplet 4 or by jumping to couplet 10 . Couplet 3 reflects an important fact. The concept of "level", while numerically being a quantity nested within the concept of what is a factor, has the interesting property as a classification character of being equal in rank to the concept of "factor". The concept of factor is itself another classification character. This equivalence leads to the fact some procedures appear more than once in the key. We could have used first the number of factors as a sorting character, and would later be faced with asking questions about the number of levels. Redundancy in the occurrence of analysis procedures would still be present.

Couplet 4. If only one factor exists, then no factorial design is possible (NFDP) and results in an extremely uninteresting experiment. The recommendation is to establish one or more additional factors, with typically two (or at times more than two) levels, to the scheme and re-apply the key. Choice "b" of this couplet directs the analyst to further criteria presented in couplet 5.

Couplet 5. Here, if only two factors exist at only two levels each, the recommendation is to add one or more levels to each factor and try to use the additive, main effects, multiplicative interaction model (AMMI). Key references are Milliken and Johnson (1989) and Gauch (1992). Milliken and Johnson (1989) present the release 5 SAS $^{\circledR 1}$ statements; one of us (JLW) has updated these statements for release 6 and a copy is available on request. A company, Microcomputer Power (111 Clover Lane, Ithaca, NY 14850 USA), provides a software package $\left(\right.$ MATMODEL $^{\circledR}$ ) that accompanies Gauch's (1992) text. A paper by Willers et. al. (1995)

\footnotetext{
${ }^{1}$ Mention of a trademark or proprietary product does not constitute a guarantee or warranty of the product by the USDA and does not imply its approval to the exclusion of other available products.
} 
describes the use of AMMI to compare the output of a deterministic soil hydrology model to replicated field data. An additional point that is suggested by this couplet and the recommendation to add more levels is the fact that doing so broadens the realm of inference. This wider horizon assists the modeler in getting more insight into the behavior and applicability of the deterministic model. The same principle applies to couplet 6 that follows.

Couplet 6. This couplet separates experiments that have three factors with only two levels each from those that have more than three factors with only two levels each. If there are only three factors with two levels in the original treatment structure, the recommendation is to add one more level to each factor and branch to couplet 12; otherwise branch to couplet 7.

Couplet 7. If there are only four, but fewer than eight, two-leveled factors, the key selects halfnormal plotting (Daniel, 1959) as the procedure to estimate the error variance. An experiment with four to six, two-level factors may not have enough information to pool non-significant highorder, interaction effects as an estimate of error (see couplet 8). Milliken and Johnson (1989) describe the use of half-normal plotting and provide several chapters describing the use of the technique. Contact the authors (JLW) for a copy to the SAS ${ }^{\circledR}$ statements that have been revised for version 6. Also, Sequeira et al. (1996) describe an example analysis that evaluates modification of the irrigation rule-base of a deterministic expert system. This example includes the interpretation of a four-way interaction. If there are more than seven, two-leveled factors, branch to couplet 8 .

Couplet 8 . If the time to complete the necessary runs is not burdensome, then one can pool nonsignificant, higher-order interactions to estimate the error (Milliken and Johnson, 1989; p. 97). For deterministic simulation experiments, we recommend the use of half-normal plotting as a graphical aid to check the assumption that higher-order interactions are "... contrasts which measure experimental error rather than effects of sampled treatment combinations (Milliken and Johnson, 1989)". As was true for half-normal plotting in couplet seven, Milliken and Johnson (1989) can be consulted for general advice on the procedure. The software to complete the analysis is quite similar to that used in couplet seven. Stevens et al. (1996) used the pooling technique and half-normal plotting to describe and interpret simulation runs of cotton production. If the computation time to complete the necessary runs is considered to be excessive, then branch to couplet 9 .

Couplet 9. This couplet, as with couplet 10, is trichotomus, rather than dichotomus, in that three choices are offered. If this is a screening design in which the number of levels has been simplified to two per factor for the purpose of identifying important factors, upon completion of its analysis by use of half-normal plotting and/or Bayes effect (i.e. Pareto) charts available with the $\mathrm{SAS}^{\circledR}$ system (e.g., ADX Menu of SAS $/ \mathrm{QC}^{\circledR}$ ), couplet 13 will instruct in the development of a more detailed multi-level fractional factorial. If the number of two-level factors does not exceed 35, then fractional factorial plans can be easily used to advantage. The ADX ${ }^{\circledR}$ system can also be used to develop a fractional factorial plan based on the theory of Hadamard matrices. 
Plackett \& Burman (1946) utilized Hadamard matrices as fractional factorial plans called saturated main-effect plans, because only $N$ runs are required to estimate all main-effects for $N-1$ two-level factors. The text by Law and Kelton (1991) discusses the use of fractional factorials and describes much of the relevant terminology. If the number of two-level factors is excessive, perhaps exceeding 35, and you suspect many of these factors will have no effect upon the response of interest, then a supersaturated fractional factorial plan can be constructed by the technique of Lin (1993). Supersaturated fractional plans require only $N$ runs to estimate all main-effects for $M-1$ two-level factors $(M>N)$, under the assumption that many of the $M-1$ factors have negligible effect on the response of interest. If the two-level factors are 15 or fewer in number, Vinyard (1989) describes a FORTRAN program based on Franklin (1985) that can be used to construct a fractional factorial design with specified main-effects and interaction effects clearly (i.e. orthogonally) estimable. If the two-level factors are 12 or fewer in number, Turiel (1988) describes a FORTRAN program, simpler in complexity than Vinyard (1989), that can be used to construct a fractional factorial design having effect estimability properties less specific than those of Vinyard (1989). Further information on the use of fractional factorials can be found by consulting Dey (1985); a text that is presently out-of-print. Dey (1985) discusses a variety of methods for constructing fractional factorial designs, many of which may be simpler than using one of these FORTRAN programs. Milliken and Johnson (1989) also discuss fractional replications of $2^{\mathrm{n}}$ factorial treatment structures. We are currently performing the analysis of deterministic mathematical program using several of the fractional factorial techniques just described here.

Couplet 10. This couplet was reached from couplet 3. If there are only one or two factors, one should branch to couplet 11 ; if there are only three factors then jump to couplet 12 . If there are more than three factors having more than three levels per factor or if the factors have a varying number of levels, then this is a design that has not yet been explored. Therefore, alternative "c" is presently classed as "CE (cutting edge)". More work is expected to be done with this latter alternative. However, for now it can be stated that Dey (1985) provides a comprehensive catalogue of fractional factorial plans and description of the various techniques for their construction. Though Dey illustrates techniques for constructing fractional factorial plans for asymmetric designs (i.e. not all factors have the same number of levels), these techniques are also useful for symmetric designs (i.e. all factors have the same number of levels) with more than three factors having more than three levels per factor. Vinyard (1989) proposes a methodology for the construction of asymmetric fractional factorial designs which utilizes Dey (1985), Franklin (1985), and the abovementioned FORTRAN program as the major components. Techniques for analysis of these designs is neither trivial nor well established. This explains why this situation is presently classed as CE (cutting edge). More work is expected to be done with this alternative. Consult a statistician for assistance with constructing your design and analyzing the resulting data.

If you are at the beginning of your experimental inquiry and it is possible to do so, you could simplify your design from a multi-leveled design as described in alternative "c" to a 
screening design. A screening design is composed of factors all having two levels with the purpose of distinguishing between important and unimportant factors relative to the response of interest. If you can simplify to a screening design; go to couplet 9 and choose from one of the three fractional factorial designs previously described.

Couplet 11. If there is only one factor, similar to $4 \mathrm{a}$, no factorial design is possible (NFDP). If there are only two factors, then apply AMMI as previously described in 5a.

Couplet 12. If all three factors are quantitative and three-leveled, then half-normal plotting, as described in $7 \mathrm{a}$, can be used to advantage. If the factors are mixed, in that some are quantitative and others are qualitative, then perhaps AMMI should be utilized. Compose the appropriate twoway treatment structure by combining two of the three factors. Milliken and Johnson (1989) provide an example analysis to choose the best film development process; however, such a combination has to be carefully done and should be done under the direction of a consulting statistician.

\section{Discussion.}

Modelers, while quantitatively skilled, are often unskilled statisticians. People who use a model are often even less skilled. Therefore, there is a need for a key, or guide, to help select the suitable analysis for a deterministic simulation experiment. The information presented in Table 1 (or Figure 1) serves this purpose. The explanation provided for each endpoint in the key also directs people to known software that can be utilized to perform and complete an analysis of their output. The software provides the benefit of helping to convey the meaning of statistical expressions and assumptions used to perform the analysis. Further, the availability of software takes much of the labor out of the analysis, so that these tools are appropriately used. Software can also encourage the modeling community to aggressively analyze their simulation experiments.

The "CE (cutting edge)" option of the key points out another benefit of the use of a key to methods of statistical analysis. A key can serve as a vehicle to tersely capture a body of knowledge about a subject area. Other researchers can build upon, and refine, or enhance a previously developed key. We hope that others will take the opportunity to refine the key presented here (or help develop methods that fill in gaps) and communicate their contributions to us. Even with these gaps, however, the procedures we describe provide a tremendous amount of insight into the performance of a deterministic simulation model.

An important element in the analysis of any experiment is estimating the error variance. An estimate of the error variance for an unreplicated, deterministic simulation experiment is useful and provides an effective tool for getting further insight on the system being simulated. The robustness is due to the fact that the estimates of error are very predictable, because the "experimental unit" responds exactly the same every time specific sets of controllable input 
variables are submitted. This fact presents a simple, yet profound point. Deterministic simulation models are unique entities in their own right, despite the assertion by Law and Kelton (1991) who quote that "...deterministic models are a special case of stochastic models... ". This historical view that places deterministic models as specialized extensions of stochastic models is not the most appropriate view, because it ignores the invariant behavior of the model as an experimental unit. As a consequence of overlooking this attribute, many modelers have tried to "force" the analysis of deterministic simulation experiments into a stochastic framework. For example, one way that replication has been tried with deterministic models was to introduce different inputs and to consider each different set of input as a replication. This process ignores the fact that replication in deterministic experiment is not possible. It is a far better view to consider each set of inputs as unique treatment combinations that are replicated only once. In summary, we propose that deterministic simulation experiments are not a specialized subset of stochastic simulation experiments. They are experiments that are as important as stochastic simulation experiments, but require the application of unique methods of analysis. One should not universally force upon them the application of statistical techniques best suited for use with stochastic models.

\section{Conclusion.}

The key adequately captures a structure that can be used to select one of many unreplicated, factorial analysis procedures useful for the analysis of deterministic simulation experiments. Adoption of the viewpoint that the deterministic model is the basic experimental unit and the inputs provided to the model constitute treatments has helped several modelers become more satisfied in how they analyze, interpret, and even verify and validate their deterministic models. Our purpose in this paper is to increase the awareness of these techniques among the community of model developers and users. The key should serve in the selection of the correct, unreplicated, factorial design and screen out those simulation experiments where these techniques are not appropriate.

\section{$\underline{\text { Acknowledgments }}$}

The authors thank Royce Bowden and Patrick Gerard of Mississippi State University, and other anonymous reviewers, for their initial review of this manuscript. Appreciation is expressed to Ron Sequeira for the preparation of Figure 1. Also, the Conferences on Applied Statistics in Agriculture, held annually at Kansas State University, has influenced and helped motivate the ideas presented here.

\section{$\underline{\text { References }}$}

Box, G. E. P., Hunter, W. G. \& Hunter, J. S. (1978). Statistics for Experimenters. An Introduction to Design, Data Analysis, and Model Building. John Wiley and Sons, Inc. New York. 
Cochran, W. G., \& Cox, G. M. (1957). Experimental Designs. John Wiley and Sons, New York.

Daniel, C. (1959). Use of half-normal plots in interpreting factorial two-level experiments. Technometrics, 1: 311-342.

Daniel, C. (1976). Applications of Statistics to Industrial Experimentation. John Wiley and Sons, Inc. New York.

Dey, A. (1985). Orthogonal Fractional Factorial Designs. John Wiley and Sons, Inc. New York.

Franklin, M. F. (1985). Selecting Defining Contrasts and Confounded Effects in $\mathrm{p}^{\mathrm{n}-\mathrm{m}}$ Factorial Experiments. Technometrics, 27: 165-172.

Garratt, M. (1975). Statistical techniques for validating computer simulation models. Tech. Rpt. 286. International Biological Program Grassland Biome: Ecosystem Analysis Studies.

Gauch, H. G., Jr. (1992). Statistical Analysis of Regional Yield Trials: Ammi Analysis of Factorial Designs. Elsevier. Amsterdam.

Law, A. M. \& Kelton, W. D. (1991). Simulation Modeling and Analysis, 2nd Edition. McGraw-Hill, Inc.

Lin, D. K. J. (1993). A New Class of Supersaturated Designs. Technometrics, 35: 28-31.

Milliken, G. A. \& Johnson, D. E. (1989). Analysis of Messy Data, Vol. 2. Nonreplicated Experiments. Van Nostrand, Reinhold. New York.

Panis, R.P., Myers, R.H. \& Houck, E.C. (1994). Combining regression diagnostics with simulation metamodels. Eur. J. Operational Res. 73: 85-94.

Plackett, R. L. \& Burman, J. P. (1946). The Design of Optimum Multifactorial Experiments, Biometrika, 33: 305-325.

Porter, C. L. (1967). Taxonomy of Flowering Plants. W. H. Freeman and Company. San Francisco.

SAS Institute Inc., $S A S / Q C^{\circledR}$ Software: Usage and Reference, Version 6, First Edition, Volume 1, Cary, NC: SAS Institute Inc., 1995. 847 pp. 
Sequeira, R. A., Willers, J. L., \& Olson, R. L. (1996). Validation of a deterministic modelbased decision support system. AI Applications 10: 1-16.

Shannon, R. (1975). Simulation: The Art and the Science. Prentice Hall, New York, NY.

Stevens, G. L., Willers, J. L., Sequeira, R. A., \& Gerard, P. D. (1996). Analysis of Deterministic Simulation Model Performance Using Non-Replicated Factorial Two-Level Experiments. Ag. Systems (in press).

Swartzman, G. L. \& Kaluzny, S.P. (1987). Simulation model evaluation. Chapter 8. p. 209251. In G. L. Swartzman and S.P. Kaluzny (ed.). Ecological Simulation Primer. Macmillan, New York.

Turiel, T. P. (1988). A Computer Program to Determine Defining Contrasts and Factor Combinations for Two-Level Fractional Factorial Designs of Resolution III, IV, and V., Journal of Quality Technology, 20: 267-272.

Vinyard, B. T. (1989). A Methodology for the Construction of Orthogonal Asymmetric Fractional Factorial Designs. A doctoral dissertation. U. of Southwestern La., Lafayette, La., $261 \mathrm{p}$.

Wallach, D. and Goffinet, B. (1989). Mean squared error of prediction as a criterion for evaluating and comparing system models. Ecol. Modelling 44: 299-306.

Willers, J. L., Wagner, T. L., Sequeira, R. A., Theseira, G. T. \& Boykin, D. L. (1995). Analysis of deterministic simulation models using methods applicable to two-way treatment structures without replication. Agron. J. 87: 478-492. 
Table 1. A key to the classification of analysis procedures applicable to the analysis of unreplicated, factorial experiments.

\section{KEY TO NON-REPLICATED FACTORIALS FOR USE WITH DETERMINISTIC SIMULATION EXPERIMENTS}

1a. Simulation model is stochastic...

Law and Kelton 1991.

1b. Simulation model is deterministic...

2a. Output of run is not unique (i.e., a trace), many observations of interest... Garratt, 1975.

2b. Output of run is unique (i.e., a point), only one observation of interest...

3a. Fewer than three (only one or two) levels per factor...

4.

$3 \mathrm{~b}$. Three or more levels per factor...

10.

4a. Only one factor, with one or two levels; or any factor with only one level...

4b. More than one factor, two levels each...

NFDP.

5.

5a. Only two factors with two levels each. Create more levels for each factor until each has three or more levels...

AMMI.

$5 b$. More than two factors with two levels each...

6.

6a. Only three factors. Create one more level until every factor has three...

$6 \mathrm{~b}$. More than three factors with two levels each...

12.

7a. Four, but fewer than eight factors with two levels...

7b. Eight or more factors with two levels...

HNP.

8.

8a. Time to complete one run not burdensome...

$\mathrm{PHO} / \mathrm{HNP}$.

8 b. Time to complete one run burdensome...

9.

9a. Number of factors moderate (LE 15) in number...

$\mathrm{FF} / \mathrm{HNP} / \mathrm{PC}$.

9b. Number of factors large (GE 15 and LE 35) in number...

$\mathrm{SFF} / \mathrm{HNP} / \mathrm{PC}$.

9c. Number of factors excessive (GT 15)... SSFF/HNP/PC. 
(Table 1 - continued)

10a. Two factors, or only one factor...

11.

10b. Three factors only...

12.

10c. More than three factors, and more than three levels (either symmetric or asymmetric) per factor...

CE.

11a. Only one factor...

NFDP.

11b. Only two factors...

AMMI.

12a. All three factors quantitative, and only three levels each...

HNP.

12b. All three factors not quantitative, and three or more levels each. Compose two-way treatment structure by combining two of the three factors...

AMMI.

Acronyms used in Key:

AMMI = Additive main effects multiplicative interaction model.

$\mathrm{CE}=$ Cutting edge.

$\mathrm{HNP}=$ Half-normal plotting.

$\mathrm{PHO} / \mathrm{HNP}=\mathrm{Pool}$ higher order interactions and use half-normal plotting to verify assumptions.

$\mathrm{PC}=$ Pareto Charts.

$\mathrm{FF}=$ Fractional Factorials

$\mathrm{SFF}=$ Saturated Fractional Factorials.

$\mathrm{SSFF}=$ Supersaturated Fractional Factorials.

NFDP = No factorial design possible; uninteresting trivial case. 


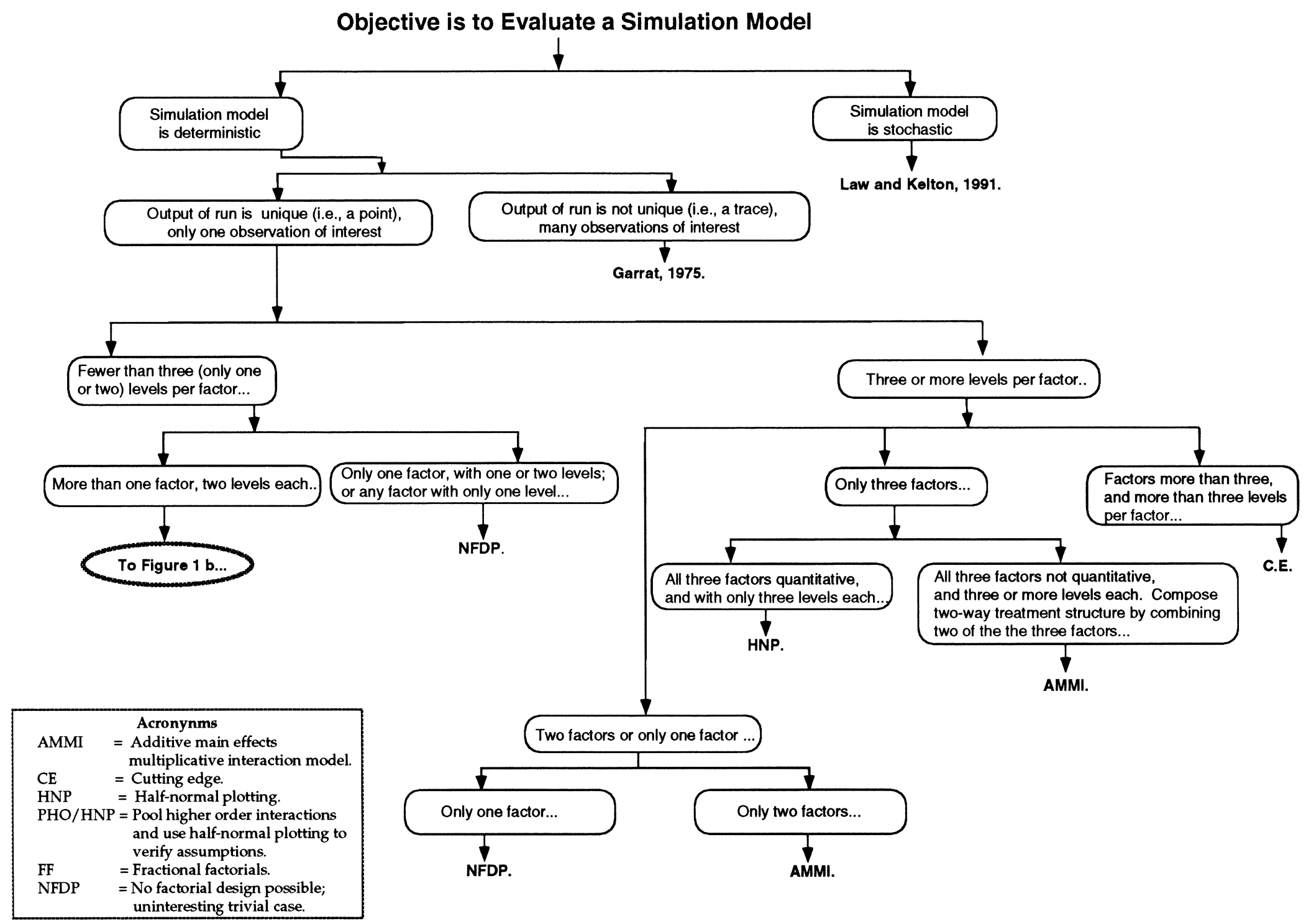

Figure 1a. Decision tree for unreplicated factorials for deterministic models. 


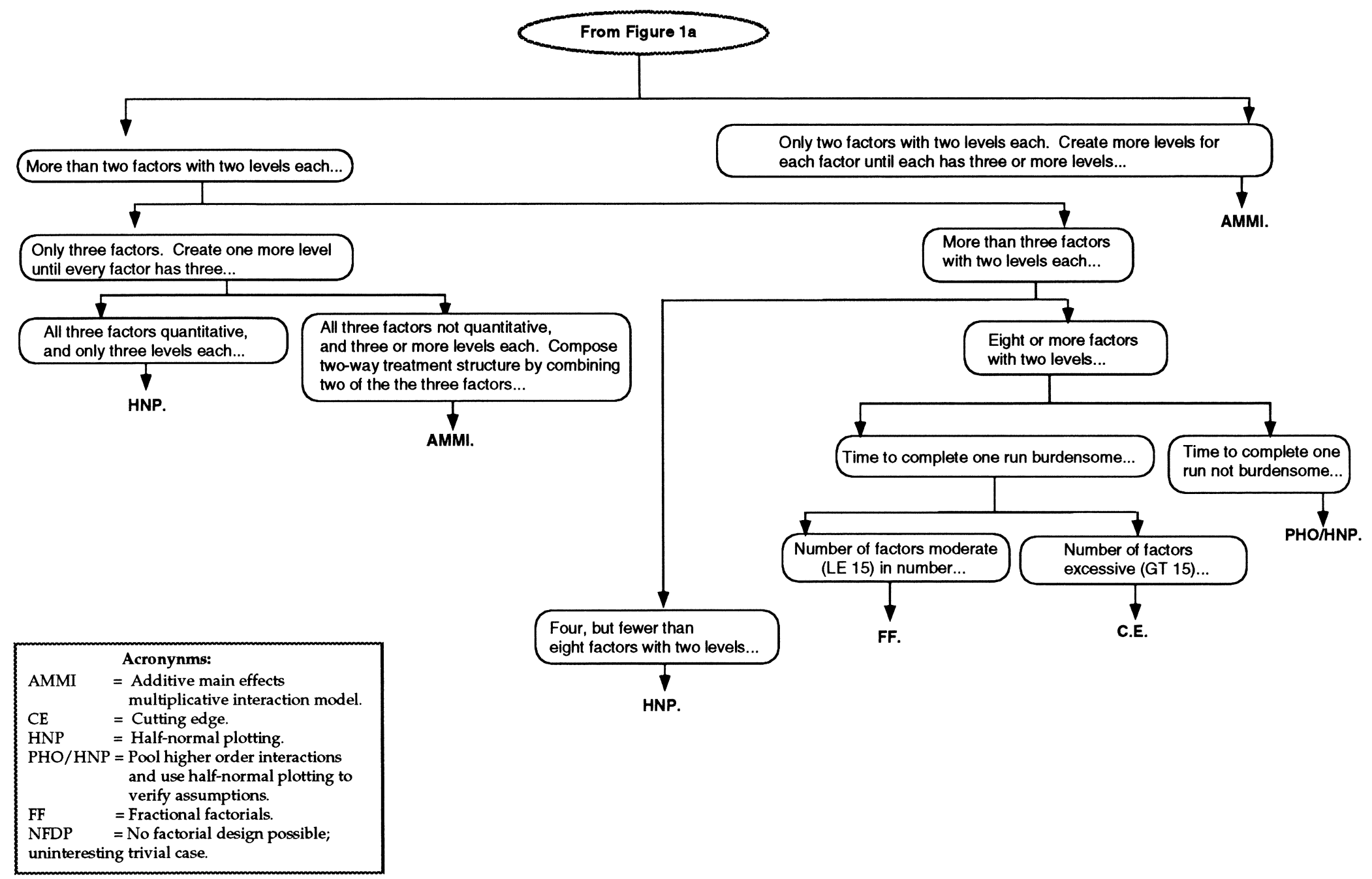

Figure 1b. Second part of decision tree for unreplicated factorials for deterministic models. 